\title{
La televisión en tiempos de streaming
}

\author{
Television in times of streaming
}

https://doi.org/10.22235/d35.2735

\author{
Juan Piñón \\ ORCID: 0000-0002-5460-7735
}

New York University, Estados Unidos

\section{RESUMEN}

El objetivo de esta presentación es el de situar los estudios de televisión en el contexto de las transformaciones tecnológicas que dieron el surgimiento de las plataformas digitales y sus posibilidades de distribución de contenido. Se inicia con una primera advertencia de no caer en determinismos tecnológicos bajo el entusiasmo de las posibilidades que la nueva ecología digital ha traído para la distribución de la televisión, para después hacer una reflexión de las tradiciones académicas que ahora dan cuenta de las nuevas modalidades de lo que constituye lo televisivo en nuestros días. Para ello se presenta una revisión breve de las perspectivas de estudio de la televisión y el surgimiento de los estudios de los medios digitales. En este último rubro, las perspectivas de estudios de infraestructura, de plataformas y de cadenas han cobrado importante relevancia, pues capturan la nueva realidad de la televisión en los tiempos de streaming. Esta presentación está basada en una revisión teórica y metodológica sobre la centralidad de la distribución y su evolución a los estudios de infraestructura (Piñón, 2018, 2019), pero también está guiada por la contribución sobre los estudios de televisión y estudios de medios digitales propuesto por Ramon Lobato (2019)..

Palabras clave: televisión; medios digitales; infraestructura; plataformas; estudios de la red.

\begin{abstract}
The goal of this presentation is to situate television studies in the context of the technological transformations which triggered the emergence of the digital platforms and their possibilities of content distribution. It starts with a warning on common assumptions from technological determinism perspectives triggered by the enthusiasm on the possibilities that the new media ecology has brought for television distribution; continuing with a reflection on the academic traditions and the way they account for the new modalities of what television is today. For this purpose, a brief survey on the perspectives of television studies and the surge of digital media studies is presented. In relation to the later, infrastructure studies, platform studies, and network studies have gained relevance because they capture the new reality of television on times of streaming. This presentation is based on a theoretical and methodological revision about the centrality of distribution, and its evolution into infrastructure studies (Piñón, 2018, 2019); but also guided by the contribution on television studies and digital media studies proposed by Ramon Lobato (2019).
\end{abstract}

Keywords: television; digital medias; infrastructure; platforms; network studies.

Nota: Esta conferencia, con el título "Los medios en tiempos de streaming", tuvo lugar el 7 de julio de 2021 en la Universidad Católica del Uruguay (UCU). La presentación se enmarcó en la inauguración del Doctorado en Comunicación de la UCU. 
Mi objetivo es hacer una revisión de algunas posibilidades que se presentan en momentos en que la industria mediática en particular ha apostado a una nueva forma de distribución de contenidos, los famosos videos on demand (VOD), que claramente han alcanzado una visibilidad enorme a través de grandes corporaciones como Netflix, Hulu, Amazon y muchas otras a nivel nacional o local.

La irrupción de estas corporaciones ha llamado la atención. Nos llevó a repensar la forma en que la oferta televisiva se organiza, se construye, se concibe y se produce. Y no es que sea un fenómeno nuevo. Simplemente el éxito de esta modalidad ha llevado a repensar toda la estructura, no solamente en televisión. Pensemos en medios y sobre algo que ha venido pasando desde hace ya tres décadas, cuyas consecuencias son ahora muy evidentes. Para ello voy a hacer una revisión que no se detiene solo en el video on demand o los servicios de VOD, sino también en pensar qué ecología mediática hace esto posible y, como pensadores de la comunicación de los medios, qué tipo de vías debemos pensar para la televisión o para los medios de acuerdo con esta nueva modalidad digital. Traigo una revisión, la cual no es comprehensiva, para presentar un panorama amplio.

\section{Nueva ecología de medios}

Una manera muy particular de entender el momento en el que nos encontramos ahora, en términos de medios, es comprender una nueva ecología de medios caracterizada por un nuevo ambiente digital que ha venido desarrollándose desde el siglo pasado con los procesos de digitalización, computarización y automatización. Estos procesos llegaron también a los medios y a casi todos los procesos de industrialización de la vida cotidiana. Esto nos obliga a pensar los medios como ambientes desde una perspectiva ecológica: su estructura, sus contenidos y su impacto en la gente; nos obliga a pensar en la mediatización que provoca este tipo de interacción, desde la materialidad misma de estos ambientes hasta la vida social que genera esta nueva modalidad.
Bajo esta idea de la ecología mediática digitalizada, computarizada, en la que estamos viviendo, hay que pensar qué es la tecnología. Y claramente muchos de ustedes ven que el sentido común nos lleva a pensar que la tecnología nos está cambiando, pero básicamente es la idea de un "determinismo tecnológico" o de una "tecnología sintomática”, como señala Raymond Williams (1990). Ambas expresiones responsabilizan a la tecnología como la provocadora del cambio social, ya sea a través de las consecuencias del desarrollo que crean nuevas sociedades o a través de una invención que accidentalmente cambia la sociedad. Bajo la idea de este determinismo tecnológico parecería que el futuro nos está llegando gracias a este desarrollo tecnológico. La idea sería, entonces, alejarnos de este discurso, pues este muestra los dispositivos mediáticos como una fuerza que estaría por fuera de las dinámicas sociales, una fuerza capaz de trascender la historia y presentarse como naturalmente irreversible y nueva. En esta predicción, los espectadores conectados son capaces de tener condiciones de conexión universal, que minimiza las reales barreras existentes en términos del orden social (Vonderau, 2014, p.102).

\section{Quitar a la tecnología como eje conceptual y volver la mirada a las practicas sociales}

Lo primero es, entonces, realizar una advertencia: hay que evitar la idea de que es la tecnología la que nos está cambiando a nosotros, una idea muy en boga gracias al nuevo ambiente digitalizado y computarizado en el que estamos viviendo. Williams (1990) nos recuerda que la tecnología es el producto de un desarrollo histórico social y que los descubrimientos tienen una intención; que el proceso de descubrimiento no es accidental ni es independiente, y que la ciencia y la tecnología se desarrollan de acuerdo con una intención muy clara de necesidades sociales y con una movilidad del desarrollo industrial que está dado y que es promovido por ciertos grupos de poder que quieren mantener al sistema tal como está funcionando. 
Este desarrollo está anclado en prácticas sociales que, muchas veces, resuelven ciertas necesidades, pero estas son muy particulares para distintos grupos de poder. 0 sea, la tecnología podría resolver cualquier necesidad, pero la necesidad tiene que ser valorada en el contexto social. Por lo tanto, no habría que caer en la tendencia de afirmar que la tecnología es la que nos está cambiando. Internet fue creado con un propósito muy evidente y ahora nos beneficiamos de él, pero todo lo que ha seguido a continuación ha tenido una raíz dentro de un proceso social e histórico. En este sentido, quiero remarcar la idea de Arjun Appadurai (1996) sobre la imaginación. Él dice:

La imagen, lo imaginado, el imaginario, estos tres términos nos llevan directo a algo crítico en los nuevos procesos culturales globales: la imaginación como práctica social. No es más fantasía simple [...], no es un simple escape [...], no es más solo un pasatiempo de las elites [...], y no es más pura contemplación [...], la imaginación se ha convertido un campo organizado de prácticas culturales, una forma de trabajo y una forma de negociación entre el sitio de la agencias (individuales) y sus posibilidades definidas en el contexto global (Appadurai, 2005, p. 31).

¿Por qué regreso a Appadurai en el sentido de la imaginación como práctica social? Porque en el terreno en el que estamos nos permite explicar lo que son las visiones del futuro. La historia de las tecnologías de la información está llena de visiones. Estas visiones están creadas por ciertos grupos y sirven a ciertos agentes para ciertos propósitos. Así, desde los sesenta hasta los setenta, el futuro ya venía siendo imaginado y propuesto. Este futuro, estas cosas que ahora usamos, ya estaban imaginadas desde entonces, como en Los Supersónicos o en las caricaturas, ya había una decisión de moverse hacia allí y así está representado en la cultura popular (Vonderau, 2014).
Una parte de esta imaginación siempre ha sido la idea de las audiencias conectadas en diferentes sitios del globo, lo que nos permiten romper las barreras de tiempo y espacio. Y así llegamos a la sala donde se mira televisión, a la venta del marketing de la sala en la que ahora vivimos, que fue imaginada con anterioridad y que no es un accidente (Vonderau, 2014). Lo importante de esta imaginación es que estamos hablando de una imaginación que opera bajo una industria y un mercado que están pugnando por nuevos consumidores y por nuevas audiencias. Entonces:

La sala está muy lejos de convertirse en un espacio donde diferentes sistemas tecnológicos, sociales o legales operan sin ningún problema. Más bien se ha convertido en un sitio para la batalla de mercados y la pérdida de lo que antes funcionaba, basada en el derecho a la exclusividad, el consumo repetido, el tiempo variable y los puntos de los precios (Vonderau, 2014, p. 102).

De alguna manera, la sala y la tecnología representan también esta lucha por mercados y las tecnologías, que continuamente están tratando de crear más mercados o de capturarnos como posibles consumidores. Esto se va complicando con más ofertas de diferentes actores que entran dentro de los que sería la operación o la interoperabilidad de lo que es hoy la sala, que es básicamente una batalla campal por el consumidor.

Entonces, pensando en esto: ¿cómo pensamos la televisión? Hay dos maneras, planteadas por Ramón Lobato (2019), de pensar en la televisión en estos momentos. Una consiste en pensar desde la perspectiva de medios digitales e Internet, que básicamente ha centrado su visión en tres niveles de estudio: la infraestructura, las plataformas y las redes en Internet. El otro es desde los estudios de televisión, los estudios tradicionales de la televisión, pero que presentan dos modalidades para entenderla: una desde momentos de disrupción de la televisión y la otra donde encontramos las continuidades de la televisión a pesar 
de entrar en otro ambiente, es decir, en un ambiente digital. Lobato propone pensar en las dos posibilidades de manera simultánea para no perder la riqueza de lo que está pasando en términos de lo que sería lo televisivo en tiempos contemporáneos.

\section{Estudios de infraestructura}

Pero voy a empezar con una visión poderosa en términos de lo que sería esta ecología, que está constituida por los estudios de infraestructura, de acuerdo con lo que, en los estudios de medios, se denomina "el giro infraestructural" (Plantin \&t Punthambeka, 2018). El objetivo no es simplemente estudiar las propiedades tecnológicas de un medio de comunicación particular, sino más bien mostrar que el transporte material de información reformula las cuestiones tradicionales de producción, circulación, acceso, consumo y política de los medios y su regulación. El transporte de comunicación se vuelve importante. ¿Por qué? Porque las infraestructuras comprenden la arquitectura de circulación, que literalmente provee la base de las sociedades modernas al generar el ambiente del entorno de la vida cotidiana. Cuando hablamos de los servicios de Internet o de los servicios de VOD, estamos hablando de toda una infraestructura subyacente: cómo llega ese servicio, quiénes tienen acceso, qué velocidad tiene. Porque existe toda una serie de formulaciones que están allí pero que son invisibles para que nosotros podamos disfrutar de nuestro sistema televisivo en el ambiente digital. Esta infraestructura también ordena la vida, así como la forma en que nos relacionamos también limita algunos accesos y permite el acceso a otros. Esto subyace en la forma que nosotros nos estamos relacionando con el medio, en este caso con el medio televisivo.

Las infraestructuras están constituidas por redes que facilitan el flujo de mercancías o ideas, y que permiten su intercambio en el espacio. Como formas físicas, ellas moldean la naturaleza de las redes, su velocidad y su dirección, sus temporalidades y su falibilidad, como es el caso de los datos de información que corren a través de grandes cables submarinos que conectan Estados Unidos con Europa, donde se da el más importante intercambio de datos a nivel global, o los cables submarinos que llevan los datos de todas las bases que unen a todo el mundo bajo el control de ciertas corporaciones y de ciertos gobiernos (Park \& Starosielski, 2015). Y si estos cables sufren algún tipo de desperfecto, evidentemente la consecuencia sería global o transnacional.

En el caso de las infraestructuras digitales de medios, el acercamiento adquiere mayor relevancia, pues tras haber "interrumpido" muchos sectores de la vida social, política y económica, muchas de las plataformas digitales más utilizadas parecen funcionar ahora como infraestructuras. Algunas de las grandes corporaciones que empezaron con una lógica de plataforma se han convertido en corporaciones de infraestructura con importantes consecuencias. No se trata solamente de cómo se mueve el contenido en el sistema, sino de cómo se ve afectado en el proceso de distribución (Plantin \& Punathambekar, 2018). La infraestructura en sí misma es también una construcción discursiva (Park \&t Starosielski, 2015). Y en la infraestructura estamos hablando de los servidores, de los cables, de los edificios, de las torres que mantienen todo este nuevo soporte de intercambio digital, es decir, la entrada del sector de computación como "distribuidores de medios" y como proveedores de contenidos.

Aquí estoy haciendo referencia a una lógica donde un sector de medios, que eran las compañías de medios protegidos por regulaciones, se ha venido diluyendo. Las empresas de telecomunicación han entrado a la lógica de medios, así como las empresas de software y digitales. Estas se han convertido en distribuidoras de medios, como Google, Facebook, Netflix, Amazon, etcétera, que son los nuevos actores dominantes y de hecho los participantes de la infraestructura misma que sostiene la red para la televisión. Esto es un dato importante, porque estas corporaciones son también plataformas que no solo ofrecen un servicio, sino que también son las propias generadoras de una infraestructura para la vida social. Así, la televisión por Internet es un espacio de oportunidades y de 
obstáculos para sus participantes, que están íntimamente relacionados con su grado de participación en la propia infraestructura de la red.

Si consideramos a Amazon, por ejemplo, esta es una de las más importantes corporaciones de infraestructura a nivel global y puede abrir negocios en particular, es decir servicios de lo televisivo, gracias a que es dueña de una gran parte de la infraestructura a nivel mundial, de los cables, de los servidores y de las posibilidades de que estas señales televisivas circulen nacional y globalmente.

En este panorama de infraestructura hay un componente de desigualdad y de poder: las infraestructuras digitales están marcadas por el encuentro poscolonial, según Miriyam Aouragh y Paula Chakravartty (2016). Ellas denuncian cómo la escala global-internacional de estas estructuras está íntimamente relacionada con la dominación material imperial, es decir, son instrumentos de vigilancia, guerra y control; están marcadas por historias de desigualdad en el contexto de un capitalismo fracturado y se han convertido en un sustento de globalización neoliberal. Claramente, la propuesta de las autoras apunta a la relación poscolonial del norte sobre el sur y a la forma en que esta infraestructura refleja esta relación muy crudamente.

\section{Estudios de plataforma}

A partir de la idea de una infraestructura que genera los ambientes donde nos movemos para acceder a los medios, hay un elemento que ha evolucionado, es decir, ciertas corporaciones de plataformas se han convertido en corporaciones de infraestructura, tales como Google, Tencent, Amazon y Facebook, que comenzaron como plataformas con objetivos y áreas específicas de operación $\mathrm{y}$ ahora parecen funcionar como infraestructuras vitales en el mundo de acuerdo con lo que Anne Helmond (2015) llama plataformización, un modelo económico e infraestructural dominante en la red digital social.

En este sentido, hay una descentralización de las funciones de las plataformas y una recentralización de platform ready desde los sitios de redes sociales hacia plataformas de redes sociales. También abre sitios web habilitando su programabilidad con una interfaz de software y una API (Interfaz de Programación de Aplicaciones) para terceros (Helmond, 2015).

La plataformización ha representado una suerte de evolución de las posibilidades que la infraestructura ha dado a ciertas corporaciones y con efectos muy reales. Como tales, las plataformas se manejan bajo un tipo de política e ideología inherentes a la manera en que se conciben y se desarrollan (Gillespie, 2010). La plataforma es el término que da forma a una política de información donde la primera busca protección para facilitar la expresión del usuario, pero también para buscar una responsabilidad limitada a lo que esos usuarios pueden decir. Tarleton Gillespie (2010) argumenta que el discurso populista a veces se presenta como plataformas desde las que se puede hablar, mientras que el discurso de marketing lo hace como plataformas de oportunidad. Así, las plataformas están diseñadas de tal manera que les permite evadir la responsabilidad de la corporación y solo se ven como un espacio para que la gente se comunique y hable. Sin embargo, evaden la responsabilidad legal sobre consecuencias que han sido evidentes en los últimos años, pero que se inscriben dentro de una idea libertaria en tanto espacios de oportunidad.

De la misma manera, "el ecosistema de plataformas determina un conjunto especifico de normas y valores inscritos en su arquitectura y que están codificados en políticas de datos, algoritmos y modelos comerciales" (Van Dijck, Poell \&t De Wall, 2018, p. 19). En otras palabras, está inscrito "un conjunto de valores libertarios donde los individuos tienen la responsabilidad primordial de organizar sus propias vidas en comunidades autoseleccionadas, con pocas obligaciones hacia la colectividad organizada por el Estado” (p. 19). ¿Por qué esto es importante? Porque la apuesta por esta ecología, por estas plataformas y por la nueva forma en que nos comunicamos determina el alejamiento de un objetivo de responsabilidad tanto del Estado como de la corporación respecto de la sociedad y donde se privilegia la idea del individuo y su libertad. 
Jean-Christophe Plantin, Carl Lagoze, Paul N. Edwards y Christian Sandvig (2018) señalan este momento como el del capitalismo tardío, es decir, cuando se empieza a destruir este contrato social. En este contexto, el ideal de la infraestructura moderna estaría desapareciendo.

La historia de los recursos informativos en red se puede ver como la transformación de un modelo de infraestructura de monopolio tradicional en un modelo de infraestructura desregulado, privatizado y fragmentado, que podríamos denominar "plataforma", que prevalece hoy en muchos sectores. Las plataformas pueden adquirir escalas enormes, coexistir con las infraestructuras y, en algunos casos suplantarlas (p. 301).

El problema es que esta noción se aleja de esta idea del Estado y de la salvaguarda de una cohesión y armonía de la sociedad en tanto se trata de entidades que buscan claramente un lucro y donde la idea de libertad del individuo, como posible consumidor, determina su lógica.

De alguna manera, existe una doble articulación en el manejo de las plataformas de parte de las empresas. "El objetivo de las empresas de recopilar datos personales de los usuarios determina las propiedades técnicas de las plataformas, lo que, a su vez, da forma a la forma en que organizan la comunicación entre los usuarios" (Plantin et al., 2018, p. 297). Esto es interesante, porque el segundo objetivo sería el negocio que reside en los datos y la forma en que se pueden recuperar estos datos, la cual da pie a la forma en que los usuarios se relacionan entre sí con el propósito, justamente, de dar más datos a la corporación. Un clásico ejemplo son las redes sociales, Facebook, Instagram o Twitter, que son las que más se manejan bajo esta lógica.

\section{Estudios de las redes}

Existe un tercer elemento dentro de la perspectiva del uso de las redes digitales que se conoce como Network Studies.
Estas explican los impactos de los medios en línea, los cuales oscilan entre las acciones de individuos empoderados y las estructuras de distribución implementadas por corporaciones poderosas. Hay una retórica de empoderamiento del usuario donde las personas controlaban el tiempo y la sustancia de sus encuentros con los medios (Wu, Tajena \& Webster, 2020, p. 2).

Estos autores dicen que toda esta idea de marketing libertaria con la que se manejan las redes es, básicamente, una ilusión. Sin embargo, de alguna manera hipotetizan la posibilidad de que, a través de las redes sociales, se genera una noción de "flujo programático" donde la forma en que se dan los clics en las diferentes redes no parece ser tan aislada o tan accidental, sino que las propias redes manejan ciertos flujos donde se podría asimilar la forma en que las propias redes generan clics o views de forma similar a la que una televisora programa su programación en cierto momento para atraer a ciertos espectadores. Los usuarios de redes pueden ser concebidos como audiencias y todo esto se genera a través de flujos de atención en lugar de flujos de audiencia. Si uno va a Twitter verá que hay trends o ciertos elementos que propone la plataforma, lo cual va generando un flujo de atención que acaba siendo algo que puede ser comparable a un flujo de audiencia (Wu et al., 2020).

El papel de las estrategias institucionales en la configuración de las secuencias de atención en la red es importante porque es equiparable a lo que podría ser un flujo televisivo. En este sentido, el estudio de Angela Xiao Wu, Harsh Tajena y James Webster (2020) trabaja mucho sobre lo que consideran las "arquitecturas de elección", las cuales influyen poderosamente en la toma de decisiones de las personas en su navegación en la web y mantienen la sensación de libertad de los usuarios al tiempo que llaman sutilmente su atención. Esto es importante porque las plataformas, como las redes, siempre manejan esta idea de libertad completa cuando en realidad hay, 
eventualmente, todo un esquema para intentar que los usuarios sigan un flujo determinado mientras la libertad que se promete, en realidad, está conducida sutilmente bajo ciertos modelos matemáticos de la propia red. Existe una secuencia de navegación que está "anclada" a un puñado de sitios que sirven como puntos de inicio y retorno en esta navegación en la red, es decir, un número limitado de constelaciones de corporaciones (Wu et al., 2020). El estudio de estas constelaciones nos habla de ciertos anclajes que permiten llegar a ciertos puntos de interés, los cuales siguen este tipo de arquitectura, que parecería semicontrolada o, por lo menos, estimulada de cierta manera para que las audiencias o los consumidores naveguen por estas estructuras.

La infraestructura, la plataforma y los estudios de redes son tres elementos con los cuales podríamos entender el concepto de la televisión desde la perspectiva de medios digitales. Y habría que sumarlos a nuestro entendimiento de lo que entendemos por "lo televisivo". Por lo tanto, ¿cómo pensar estos sistemas de streaming desde la forma en que tradicionalmente hemos pensado la televisión? Como fue comentado, hay dos maneras de entender dónde se piensa este tipo de plataformas, es decir, como anclado a este cambio o como continuidad de la televisión: cómo Netflix se parece o no a la televisión, cómo Disney Plus recobra o no elementos de lo que consideramos televisión. A esto se suma la perspectiva de medios digitales mencionada a través de los conceptos de infraestructura, plataforma y redes.

\section{La televisión se transforma a sí misma}

Ramón Lobato (2019) propone pensar la televisión más allá de considerar cómo Netflix permite o no la continuidad de la televisión. Lobato propone pensar en este modelo de acuerdo con una transformación de adentro hacia afuera, es decir, desde que empezó a digitalizarse en los ochenta dentro de un sistema de distribución en televisión que empezó a digitalizarse. Existe todo un proceso de cambio inherente a la práctica de producción televisiva que involucra toda una serie de cambios en términos de una ecología de medios y de acuerdo con una posibilidad de trasmitir o subir televisión de diferente manera, que sigue como televisión pero que genera otra cosa, como un parásito que se apodera de su anfitrión dejando intactas las apariencias externas mientras vacía los cimientos, tanto tecnológicos como ontológicos, en el que se basó un determinado medio o modo de representación (Elsaesser \& Hagener 2015, pp. 204-205).

La transformación de adentro hacia afuera de la televisión por Internet permite que la experiencia de la televisión se mantenga consistente con las normas establecidas mientras se despliega un cambio sustancial en el interior, específicamente la no linealidad y la interactividad inherentes de la plataforma de video digital. En este sentido, quiero remarcar un elemento claramente disruptivo dentro de lo que hoy entendemos como televisión a partir del streaming: la nueva forma de distribución. Y una de las ausencias dentro del estudio de comunicación y medios dentro del clásico "círculo de la cultura" es la ausencia de la distribución. Tenemos a la representación, la identidad, la producción, el consumo y la regulación, pero no está la infraestructura. La infraestructura entonces se ha vuelto un espacio importante para repensar las nuevas dinámicas mediáticas.

\section{La distribución como eje conceptual}

Timothy Havens (2002) afirma que Stuart Hall falla en no tomar en cuenta la distribución como un momento significativo entre producción y consumo donde es creado el valor económico del producto audiovisual (p. 379). Cuando hablamos de distribución no solo hablamos de la posibilidad tecnológica de distribuir sino también de las dinámicas inherentes a nivel social o a nivel corporativo para que suceda esta distribución. Esto quiere decir que los ejecutivos, en el mercado de la televisión mundial, en su calidad de "intermediarios culturales," determinan el tipo de programación internacional a la que pueden acceder las audiencias, así como el tipo de significados 
y placeres disponibles para ellas. En este sentido, a nivel local o nacional, los ejecutivos del mercado de televisión son quienes siguen decidiendo lo que vemos. Y constituyen la parte central de la distribución. Son quienes están en el medio de la producción y el consumo, quienes deciden qué se distribuye, así como los medios o las formas en las que se distribuye. A estos les ha llamado Pierre Bourdieu (1984) "intermediarios culturales".

En este proceso, un significado se produce a través de diferentes prácticas de gestión, gerenciales y de negocios, de técnicas de mercadeo, de publicidad y de relaciones públicas, las cuales se han convertido en procesos centrales en la comercialización de productos simbólicos (Negus, 2002, p. 504). Esto es perfectamente aplicable a cualquier servicio de streaming. ¿Cuáles son las decisiones de los ejecutivos para realizar una serie o para pagar los derechos de cierto contenido a nivel trasnacional, global o regional? Siguen siendo los intermediarios culturales, de estos nuevos servicios, los que determinan el valor de la oferta que ellos proponen y quienes, en sus prácticas gerenciales, incorporan a la oferta una modalidad de valor simbólico, y económico.

Amanda Lotz $(2007,2017)$ ha venido proveyendo una especie de tipificación del desarrollo de la televisión en Estados Unidos. Ella habla del arraigo de lo que sería la televisión distribuida por Internet después de cierta fase y delimita a la televisión distribuida por Internet como una subcategoría del video distribuido por Internet (Lotz, 2017). De modo que una categoría más amplia del video abarca las películas distribuidas por Internet. Una amplia gama de videos producidos fuera de las estructuras industriales puede circular dentro de estas nuevas posibilidades de distribución por Internet. Porque no todo lo que es video en Internet es televisión. En este caso, serían televisión los portales en línea que distribuyen series producidas de acuerdo con las prácticas industriales profesionalizadas de la televisión.

Con esto regreso a la idea de Lobato sobre la transformación de adentro hacia afuera: todo cambió, toda la distribución cambió, la temporalidad cambió, pero el disfrute de la oferta de lo que nosotros hemos conocido siempre como televisión sigue ahí. Yo veo, en Netflix o en el streaming que me parezca más conveniente, un programa de televisión que yo disfruto como lo disfruté siempre en términos de televisión, en términos de cómo veo televisión. Claramente, muchos de ustedes estarán pensando: "bueno, no es igual”, por muchas razones que son muy válidas. Entonces estamos hablando en una coexistencia. Y esto habla un poco más de cómo esta nueva manera de ver televisión tiene un tipo de impacto y de consecuencia, una nueva manera que se puede llamar televisión, porque las series que se producen dentro de estos formatos siguen estando ligadas a las prácticas institucionales de los profesionales de la televisión, no de los funcionarios del Internet. 0 sea, la televisión sigue viva y se produce más más televisión que nunca en la historia del medio.

La televisión sería una de todas las posibilidades de video que se ofrece dentro del Internet. Y centrados particularmente en esta modalidad, hay muchas cosas que se pueden analizar, discutir e investigar sobre la forma en que la oferta y la distribución dentro del streaming impacta en la forma en que nosotros concebimos la televisión, desde la concepción del catálogo, la función del algoritmo, la colección de datos o data mining, las audiencias y la forma en que la ven, así como las redes sociales y los servicios, la producción nacional e internacional, el tipo de regulaciones y el tipo de flujos transnacionales. Estas son algunas de las muchas posibilidades de análisis que nos ofrece el nuevo campo en términos de streaming. Uno de ellos, y que ha roto claramente con la oferta en términos de cómo concebíamos la televisión, ha sido el catálogo de los servicios de streaming.

\section{El flow de la televisión vs el streaming de los contenidos televisivos}

¿Cómo pensábamos la programación de la televisión lineal? Estaba caracterizada por una limitación en su capacidad y una especificad temporal. Solo se podía tener al aire una cosa y dada la cantidad de tiempo disponible, no se podía ver todo, no había manera ni había espacio donde poner una enorme cantidad de contenido. La idea 
de la escasez del contenido siempre había sido lo que privilegiaba la televisión y su forma de ver de manera instantánea. Hay escasez de contenido y por lo tanto hay que ver lo que está en la tele, por así decirlo. Como señala Raymond Williams (1990), se trataba más acerca del flow de la programación que de cierto programa en particular. Uno veía la tele, pero uno no veía un programa: veía la televisión. Claramente este modelo, la manera en que sustituía a este flow de contenidos, como bien lo comenta Williams (1990), estaba basado en una programación donde la publicidad era parte de este plus, era parte de ver televisión y esto sostenía al medio. En los servicios digitales de streaming todo esto queda totalmente fuera. La limitación de tiempo y la limitación de espacio no existen "como tales", y lo pongo entre comillas porque, si existe una limitación, no lo es en los términos en que se veía la televisión.

En el caso de los servicios de streaming, la estrategia está basada en una estrategia de audiencia (a quién hay que llegar) y una estrategia de adquisición de contenido, ligada a la audiencia a la que quieren llegar. Esta estrategia está muy vinculada con la focalización de esa audiencia a través de cierto tipo de tweets en los catálogos o en el algoritmo y la importancia de la marca del canal, que está ligada, a su vez y en cierta medida, a lo que tradicionalmente es la marca de canal o el branding. La focalización de la audiencia tiene un elemento importante donde los algoritmos forman parte de todo este aparato permitiendo establecer rutas de descubrimiento que solo corresponden a ciertas audiencias que están siguiendo ciertos tipos de patrones de consumo.

\section{Catálogo, algoritmo y colonialismo digital}

Tenemos así entonces un catálogo que, como afirma Lobato (2019), emula un poco una noción performativa donde, por ejemplo, Netflix siempre se ha considerado un medio digital, pero en sus relaciones públicas se nombra o se llama televisión. En otras palabras, se trata de un medio que, al mismo tiempo, tiene la lógica de catálogo pero la forma en que lo vemos se parece más bien a la forma de ver ciertas cintas de cine. En este caso es Netflix, porque ha llamado mucho la atención por su visibilidad global. Pero cualquier otro tipo de catálogo presenta una performatividad que permite atraer a cierto tipo de cliente o de consumidor.

El catálogo está claramente dominado por un algoritmo que permite descubrir ciertas cosas o no descubrirlas y está relacionado con la nueva modalidad de esta ecología de la regulación y análisis de datos. Así como el ambiente mediático crece y se hace más complejo, así como las audiencias se fragmentan y se empoderan cada vez más con una creciente variedad de tecnologías y de plataformas a su disposición, las organizaciones de medios, también manera más creciente, están volviendo al big data y a los algoritmos para ayudar en la efectividad al navegar en este panorama complejo. La recolección de análisis de datos no solo es parte del streaming, sino también de las redes.

La plataformización de la industria está muy ligada a la matemática del algoritmo, la cual tiene también sus propias consecuencias. Una de ellas, desde la teoría crítica, es lo que sería un colonialismo de datos. Existe una crítica a la recolección de datos, que se ve como natural en el contexto del flujo de la vida cotidiana. Se ha normalizado el hecho de que las corporaciones exploten los datos que producen los individuos a través de sus interacciones en la vida cotidiana. Esta normalización está anclada a una racionalidad extractiva donde básicamente se piensa que los datos están ahí, que se producen y que uno los puede recoger, lo cual emula las dinámicas del colonialismo donde los datos están ahí para ser tomados con la ventaja de que, básicamente, la gente lo permite porque no habría manera de evitar esta extracción (Couldry \&t Mejías, 2019).

Lo problemático es que, claramente, la gente está involucrada a través una plataformización donde la gente puede usar datos y, al mismo tiempo, definir la forma en que se gratifican las relaciones que la gente emplea en la plataforma. En otras palabras, la gente está produciendo los datos que la propia empresa está buscando y los cuales 
va a estructurar. El problema es que las corporaciones que están enfocadas y que pueden hacer este tipo de operaciones son de 0ccidente. 0 sea, hay una dependencia y una visibilidad enormes de poblaciones, tanto norteamericanas como europeas, chinas y japonesas, que tienen un dominio sobre este tipo de lógicas computacionales donde, en esta perspectiva postcolonial, el sur no ha tenido la capacidad de recolectar los datos como lo hacen corporaciones como Google, Facebook, Amazon, etcétera, y donde las redes sociales tienen un importante papel (Couldry \& Mejías, 2019).

En este sentido, el colonialismo de datos combina las prácticas extractivas depredadoras del colonialismo histórico con métodos abstractos de cuantificación de la computación. Comprender el big data del Sur global significa comprender la dependencia actual del capitalismo de este nuevo tipo de aprobación que funciona en todos los puntos del espacio donde las personas y las cosas están conectadas a las infraestructuras actuales de conexión (Couldry \& Mejías, 2019). Estas infraestructuras son dominadas por cierto tipo de corporaciones y entonces regresamos a la idea del imperio e infraestructura y a la crítica sobre esta dependencia.

\section{El algoritmo y la creación en la producción de televisión}

La recolección de datos tiene un impacto claro en el caso del streaming en dos áreas fundamentales: la producción y el consumo. El rol de los agentes humanos en recomendar y compartir objetos culturales está siendo reemplazado por las máquinas. Se sabe muy poco del trabajo que examina los algoritmos como diferentes tipos de conocimiento que afectan las prácticas culturales existentes incluyendo las prácticas de producción. De regreso a la producción y pensando en televisión, dos de las funciones primarias que los algoritmos realizan actualmente en la producción de medios son, en primer lugar, la predicción de la demanda y, en segundo lugar, como posibles creadores de contenido. Esto no quiere decir que el elemento hu- mano haya sido eliminado de la creación de contenido. Los algoritmos son creaciones humanas y existe un rol humano en el contexto de la creación, que ha migrado de un rol directo a uno indirecto. Los algoritmos dan el pie para lo que se debería producir de acuerdo con una posible proyección (Napoli, 2014).

En este sentido, Timothy Havens (2014) hace un análisis de la creación del primer programa de Netflix, que fue House of Cards, y plantea la forma en que el algoritmo sirvió a la creación de algo que se convirtió en un éxito. En su crítica, Havens reconoce que los algoritmos dieron a la compañía una serie de inputs acerca de lo que la gente podía ver, pero cuando el programa ya estaba realizado, es decir, los algoritmos no crearon el programa. Se trataba de un programa decidido y hecho. Simplemente hubo una apuesta de Netflix al programa porque, de alguna manera, era acorde a la información de la empresa sobre lo que podía ser exitoso. Esto es muy importante: el algoritmo no creó el programa, sino que le dio el input a un ejecutivo para decidir lo que este ejecutivo consideraba que sería el programa. Aquí regreso entonces a la noción de intermediarios culturales. El algoritmo no decide, sino que existe como una creación matemática donde, finalmente, hay un ingeniero que decide cómo realizarlo o un ejecutivo que toma una decisión sobre lo que se debería producir.

En este sentido, Havens hace una crítica importante a la idea de que los algoritmos crean la producción. Por un lado, plantea la falta de creatividad de House of Cards en términos de inversión e innovación y piensa que la parte más creativa del programa fue cuando Kevin Spacey le hablaba directamente a la cámara. Para la cantidad de dinero y para toda la capacidad tecnológica que hay invertidas en el programa, parecería que la creatividad no fuera lo más fuerte, a pesar de que haya sido claramente un programa exitoso. Pero entonces es interesante e importante, dentro de la discusión del algoritmo de la producción, el reclamo de que el algoritmo va a reproducir lo que la gente ya está viendo, por lo cual la predicción de lo que la gente podría ver está alineada a lo que la gente ya ve. 
Existe todo un debate acerca de un algoritmo que solo llevaría justamente a la pérdida de la creatividad y de la innovación. Esto es una cuestión que puede ser muy debatible, pero que nos dice que el algoritmo no hace todo el trabajo. Y cuando encontramos una serie o un producto que puede ser innovador, esta innovación no necesariamente proviene de la operación del algoritmo sino, eventualmente, de la decisión de un ejecutivo que vio una oportunidad. Por lo tanto, el algoritmo nos estaría dando algo de acuerdo con lo establecido de lo que ya se ve, las posibilidades dentro lo que ya existe, pero no de lo que podría ser.

Mareike Jenner (2016) plantea la existencia de un "efecto Netflix". En términos industriales, este efecto ha hecho que el streaming permita la existencia de más series de televisión usando el esquema serializado y bajo un esquema que llama "narrativa compleja”. Esta narrativa está ligada a la posibilidad de que las audiencias puedan ver diferentes programas de corrido, lo que se conoce como binge-watching. De esta forma, pueden hacer conexiones que no esperarian hacer al ver la serie una, dos o tres semanas después, es decir, mientras la serie se produce. Se puede ver la serie como leyendo un libro, por decirlo así, y se pueden hacer conexiones más complejas dentro de la ficción. Aunado al hecho de que las temporadas son ahora más cortas (de 10 a 12 capítulos en lugar de 24), se privilegian las grandes producciones que ofrezcan el efecto binge-watching. Se presenta entonces toda una forma de entender la televisión que consiste en entregar una serie como si fuera un libro, en capitulos que la gente va a poder ver de una sola sentada y que le permite, de alguna manera, una comprensión diferente respecto a los tiempos de lo que era la televisión tradicional.

Las series, al no haber publicidad, se manejan bajo el branding o el product placement al interior de las series de manera muy sutil. Y aquí el planteo de Jenner (2016) es interesante porque se vincula con la noción de flujo televisivo de Williams (1990) donde los comerciales existían en un flujo junto con los contenidos al mirar televisión. Parecería entonces que se reproduce una integración de las marcas de acuerdo con el product placement dentro de series donde se pueden tener diez horas de binge-watching mientras todas las marcas están allí, por lo cual se termina viendo todos los comerciales incluidos en la trama de acuerdo con la finalidad del publicista, quien ubica la marca dentro de la serie.

\section{El algoritmo, el binge-watching y la recepción de contenidos televisivos}

Para cerrar, hablaremos de los algoritmos y la recepción. Los algoritmos han permitido el consumo televisivo dentro del streaming y que las televisoras pierdan el control sobre el momento del consumo. Ahí está la oferta y es la audiencia quien decide en qué momento y cuánto va a ver. El espectador se siente empoderado y toma el control. Hay un menú para elegir, concebido en una acción racional, que es más una acción de transacción de mercado y donde la oferta está guiada por las sugerencias del algoritmo. El consumidor raramente accede a toda la oferta, sino a una ventana restringida y ligada a su historial de consumo.

En este sentido, el hecho de que un algoritmo pueda definir o no lo que uno quiera, permite pensar en lo que algunos investigadores de la Universidad de Costa Rica llaman una "domesticación mutua" entre el algoritmo y el consumidor (Siles, Espinoza, Naranjo \&t Tristán, 2019). En este sentido, explican, hay una domesticación que entienden como un proceso cíclico. Los algoritmos participan de la creación y del mantenimiento de la cultura contemporáneas, es decir, de las "formas particulares de vida, que expresan ciertos significados y valores no solo en el arte y el aprendizaje sino también en las instituciones y comportamiento" (Williams en Siles et al., 2019).

Además, los algoritmos no funcionan completamente solos: necesitan ser implementados culturalmente a medida que se incorporan a la vida diaria (Seaver en Siles et al., 2019). Por lo tanto, es necesario tener en cuenta no solo que los algoritmos dan forma a la cultura, sino también que los algoritmos, en sí mismos, son la cultura 
(Siles et al., 2019). En este sentido, los autores proponen toda una serie de relaciones con los algoritmos donde el usuario personaliza su participación en la plataforma, integra cierta posibilidad de contenidos en el contexto de rituales que les son sociales e individuales de acuerdo con sus posibilidades de vida, resiste ciertas cosas que la plataforma le obliga -ya que hay ciertos niveles de resistencia- o convierte ciertas prácticas para su conveniencia. La audiencia no es pasiva y puede eventualmente domesticar hasta cierto punto a los algoritmos.

La televisión había sido concebida siempre como la "caja idiota". Pero ahora la televisión se ubica en el esquema del binge-watching, que recupera la idea de un texto narrativo más complejo que se está definiendo dentro del esquema de streaming como un consumo de alta cultura. La práctica del binge-watching implica no solo el deseo de los espectadores de tener autonomía para programar cuándo quieren ver, sino también el deseo de un texto "puro" que no sea parte distintiva del flujo televisivo. El diseño televisivo de acuerdo con las prácticas de consumo de binge-watching parece estar ligado a estrategias más complejas de narración colocándolas en un contexto de "alta cultura". Se trata de estrategias de prácticas individualizadas y de auto programación de televisión que forman parte de una lógica del capitalismo postmoderno (Jenner, 2016).

De esta forma, el binge-watching ya no es leído en términos de "pérdida de tiempo", sino en términos culturales, como sería leer una gran novela que ofrece un cierto tipo de estatus. En este contexto, las redes sociales cumplen un factor importantísimo, porque la gente sigue compartiendo a través de las redes y discute lo que ve, aunque no sea en el tiempo real como en la televisión, pero sigue siendo un momento real donde las corporaciones y las redes permiten toda una serie de intercambios y comentarios que se ha denominado el water cooler de la conversación, donde la gente tuitea y comparte, y donde particularmente los jóvenes tienen una dinámica muy anclada en compartir sus gustos y sus placeres al ver televisión.

\section{El catálogo y los flujos de televisión en mercados transnacionales}

Existe toda una discusión sobre si el streaming y los servicios globales están creando diferentes flujos a nivel mediático global. Esta discusión tendría que ver con la idea de que, eventualmente, existía un dominio de ciertos países sobre el resto del mundo en términos de un flujo denominado one way, una calle solamente de ida. Pero parecería que Netflix, Amazon o Disney podrían estar abriendo la posibilidad de que existiera una audiencia más cosmopolita en términos de cómo se ven estos tipos de contenidos. En este sentido, habría que entender mejor cómo está conformado el catálogo a nivel trasnacional, lo que Lobato llamaría "catálogos diferenciados", pues no se trata de un solo catálogo, sino que son catálogos diferenciados temporal y espacialmente, ya que cambian constantemente y porque los usuarios, en diferentes países, experimentan diferentes rangos de contenido (Lobato, 2019). Es más preciso, según Lobato, hablar de una serie de servicios nacionales que están ligados por una arquitectura de plataforma común.

El catálogo no es la audiencia. Los catálogos diferenciados por países tienen algo que es común, tal vez definido por el algoritmo, que es, en gran medida, la oferta de la casa distribuidora, en este caso las grandes corporaciones de streaming. El algoritmo hace mucho más visible la oferta, en este caso norteamericana, británica, europea o del noreste de Asia, y prevalece sobre las ofertas nacionales. La forma en que el algoritmo ofrece la plataforma privilegia cierto tipo de contenidos (Lobato, 2019).

Hay toda una discusión en Europa sobre si el algoritmo privilegia la producción europea cuando la plataforma está en Europa, así como podría pensarse si el algoritmo privilegia lo latinoamericano si está en Latinoamérica. Se trata de una discusión que tiene mucho que ver con la regulación y las legislaciones nacionales, donde se establecen ciertas cuotas para la presencia de producciones nacionales que, en este caso, el servicio de 
streaming debería ofrecer dependiendo de la legislación. Sin embargo, aunque la cuota esté cumplida, el algoritmo no necesariamente da mayor visibilidad. Entonces cuota y porcentaje no va a significar una visibilidad en términos de lo que el presenta el catálogo (Lobato, 2019).

\section{Referencias}

Aouragh, M., \&t Chakravartty, P. (2016). Infrastructures of Empire. Media, Culture and Society, 38(4), 559-575.

Appadurai, A. (2005). Modernity at large. Cultural dimensions of globalization. (7a edición). Minneapolis, MN: University of Minnesota Press.

Bourdieu, P. (1984). Distinction. A Social Critique of the Judgement of Taste. Cambridge, MA: Harvard University Press.

Couldry, N., \& Mejías, U. (2019). Data Colonialism: Rethinking Big Data's Relation to the Contemporary Subject. Television \& New Media, 20(4), 336-349.

Elsaesser,T., \&t Hagener, M. (2015). Film theory and introduction to the senses. ( $2^{\circ}$ Edición). Londres, Reino Unido: Routledge.

Havens, T. (2002). "It's still a white world out there": the interplay of culture and economics in international television trade. Critical Studies in Media Communication, 19(4), 377-397.

Havens, T. (2014). Media Programming in an Era of Big Data. Media Industries Journal, 1(2). doi:10.3998/mij.15031809.0001.202

Helmond, A. (2015). The Platformization of the Web: Making Web Data Platform Ready. Social Media + Society, 1(2), 1-11.

Gillespie, T. (2010). The politics of 'platforms'. New Media \&t Society, 12(3), 347-364.

Jenner, M. (2016). Is this TVIV? On Netflix, TVIII and binge-watching. New Media \& Society, 18(2), 257-273.

Napoli, N. (2014). On automation in media industries: Integrating algorithmic media production into media industries scholarship. Media Industries Journal, 1(1), 33-38.

Negus, K. (2002). The work of cultural intermediaries and the enduring distance between production and consumption. Cultural Studies, 16(4), 501-515.

Lotz, A. (2007). Television will be revolutionized. New York, NY: NYU Press.

Lotz, A. (2017). Portals: A treatise on Internet-distributed television. Ann Harbor, MI: Michigan Publishing University of Michigan. doi:10.3998/mpub.9699689
Lobato, R. (2019). What is Netflix. En Netflix Nations. The Geography of Digital Distribution (pp. 19-46). New York, NY: NYU Press.

Park, L., \& Starosielski, N. (2015). Introduction. En Signal Traffic. Critical Studies of Media Infrastructures (pp. 2- 27). Urbana, IL: University of Illinois Press.

Piñón, J. (2018, agosto). This is not the end, but a new beginning for television. Presentado en el XIII International Seminar of the Ibero-American Television Fiction Observatory (OBITEL). Universidad de Sao Paulo, Sao Paulo, Brasil.

Piñón, J. (2019). USA: Network infrastructure of the internet television industry. En M. Immacolata \&t G. Orozco (Eds.), Television distribution models by the Internet: Actors technologies, strategies. Ibero American Observatory of Television Fiction OBITEL (pp. 307-340). Globo Universidade, Editora Sulina.

Plantin, J.C., Lagoze, C., Edwards, P. N., \&t Sandvig, C. (2018). Infrastructure studies meet platform studies in the age of Google and Facebook. New Media \&tSociety, 20(1), 293-310. doi:10.1177/1461444816661553

Plantin, J.C., \& Punthambekar, A. (2018). Digital media infrastructures: pipes, platforms, and politics. Media, Culture \& Society, 41(2), 163-174.

Siles, I., Espinoza, J., Naranjo, A., \&t Tristán, M.F. (2019). The mutual domestication of uses and algorithm recommendations on Netflix. Communication, Culture, and Critique, 12, 499-518.

Van Dijck, J., Poell, T., \& de Waal, M. (2018). The platform society. New York, NY: Oxford University Press.

Vonderau, P. (2014). Beyond piracy. Understanding digital markets. En J. Holt and K. Sanson (Eds.), Connected viewing. Selling, streaming and sharing media in the digital age (pp. 99-122). New York, NY: Routledge.

Williams, R. (1990). In Television. Technology and Cultural Form (2 $2^{\text {nd }}$ ed.). New York, NY: Routledge.

Wu, A., Taneja, H., \&t Webster, J. (2020). Going with the flow: Nudging attention online. New Media \& Society, 23(10), 2979-2998.

Contribución autoral

a) Concepción y diseño del trabajo; b) Adquisición de datos; c) Análisis e interpretación de datos; d) Redacción del manuscrito; e) revisión crítica del manuscrito.

J. P. ha contribuido en $a, b, c, d$, e.

Editor responsable: L. D. 